\title{
Use of opioid analgesics for the treatment of chronic noncancer pain - A consensus statement and guidelines from the Canadian Pain Society
}

SUMMARY

1

Pain of all types is undertreated in our society. The pediatric and geriatric populations are especially at risk for undertreatment. Physicians' fears of using opioid therapy, and the fears of other health professionals, contribute to this problem.

\section{2}

Chronic noncancer pain (CNCP) is generally defined as pain lasting at least six months; or of a duration longer than the expected time to tissue healing or resolution of the underlying disease process; or due to a condition where there is ongoing nociception. CNCP is different from acute pain in both its presentation and pathophysiology. Progress in basic science research is gradually discovering the biochemical and structural mechanisms of peripheral and central sensitization that maintain chronic pain. It is, therefore, possible that more specific treatments will become available in the future.

\section{3}

Patients with CNCP require a thorough assessment before deciding on treatment. A patient with chronic pain may have physical, psychological, social and/or behavioural contributors to suffering that may require specific attention in a comprehensive treatment plan.
The Canadian Pain Society task force that developed this consensus statement and guidelines consisted of Roman D Jovey MD (Chair), Jeffrey Ennis MD FRCPC, Jacqueline Gardner-Nix MBBS PhD MRCP(UK), Brian Goldman MD FACEP MCFP(EM), Helen Hays MD CCFP, Mary Lynch MD FRCPC and Dwight Moulin MD FRCPC.

Review and suggestions were also provided by A John Clark MD, Allen Finley MD, Neil Hagen MD, Sandra LeFort RN $\mathrm{PhD}$, Harold Merskey DM and Russell K Portenoy MD.

4

Many treatment options exist for CNCP, including physical, psychological, pharmacological and surgical options. In the absence of good evidence for a specific, curative treatment for a given pain problem, a trial of long term opioid therapy is a legitimate medical practice when a reasonable trial of other standard treatment modalities fails to improve comfort or function for the patient. There are few types of pain that preclude a trial of opioid therapy.

\section{5}

Tolerance and/or physical dependence on regular opioid use in a patient in pain is not, by itself, evidence of an addictive disorder. Addiction is a biopsychosocial disorder character-

This consensus statement has been adapted in part from two existing statements: "The use of opioids for the treatment of chronic pain: A consensus statement from the American Academy of Pain Medicine and the American Pain Society" (1997); and "Use of opioid analgesics for the treatment of chronic pain of nonmalignant origin: A discussion paper from the Probationary Section on Chronic Pain of the Ontario Medical Association" (1997)

This document was approved by the Executive of the Canadian Pain Society on November 18, 1998

Correspondence and reprints: Canadian Pain Society, Attention: Joan Hoskins, 50 Driveway, Ottawa, Ontario K2P 1E4. Telephone 613-234-0812, fax613-234-9894,e-mail cps@is.dal.ca 
ized by the compulsive use of a substance and preoccupation with obtaining it, despite evidence that its continued use results in physical, emotional, social or economic harm. A patient with a past history of or risk factors for addiction should not necessarily be precluded from a careful trial of opioid therapy, although in such a case consultant advice might be sought.

\section{6}

Opioid analgesics are generally safe medications when prescribed with appropriate monitoring. There is very little if any evidence of organ damage from the long term therapeutic use of opioids. With appropriate titration and stable dosing, tolerance develops to most of the side effects of opioid therapy, including cognitive impairment. Constipation is the most common persistent side effect and should be managed prophylactically.

\section{7}

A key principle in the treatment of all types of pain with opioids is dosing to effect or to the point of persistent and unacceptable side effects. In patients with around-theclock pain, opioids should be dosed in a pharmacologically appropriate, time-contingent schedule, rather than a PRN dosing regimen. The use of an opioid analgesic with a long duration of action can improve patient compliance thus facilitating better tolerance to side effects such as cognitive impairment, and may reduce the fluctuation in pain based on PRN dosing regimens. In the opioid-naive patient, failure to realize at least partial analgesia with incremental dose titration may indicate that the pain syndrome is unresponsive to opioid therapy; however, in some patients with more severe pain problems, significant analgesia may only occur after a threshold dose of opioid has been reached.

\section{8}

The goal of long term opioid therapy is improved quality of life for the patient in pain. This improvement should include, as a minimum, a significant decrease in pain severity and ideally an improvement in physical, psychological, social and occupational functioning. The patient on long term opioids needs to be reassessed periodically to ensure an ongoing benefit of treatment. Physicians should carefully reassess a patient who demonstrates repeated episodes of aberrant drug-related behaviour or whose function declines as a result of opioid therapy. Extra caution should be exercised in such cases and it may be appropriate to reconsider the use of the drug, tapering it off before discontinuation.

\section{9}

The use of long term opioid therapy in CNCP does not preclude the concurrent use of other treatments such as nonopioid analgesics, or physical or psychobehavioural modalities. However, the use of any type of sedative medication that may also cause additive, long term cognitive impairment should be avoided if possible.
10

Adequate documentation is essential to demonstrate the evaluation process, including consultations and relevant investigations, the rationale for long term opioid therapy in the context of the overall management plan and the periodic review of patient status. In addition, documentation is required to demonstrate compliance with federal controlled substance legislation.

\section{GUIDELINES}

\section{I) Definition of chronic noncancer pain}

1: Chronic noncancer pain (CNCP) is defined as noncancerrelated pain with a duration longer than the expected time to tissue healing or resolution of the underlying disease process. $\mathrm{CNCP}$ can also be due to a condition where there is ongoing nociception, such as the inflammation of various arthritic conditions. Other previously used terms for this type of pain include chronic nonmalignant pain, chronic pain of nonmalignant origin and chronic benign pain. Autonomic features suggestive of acute pain, such as anxiety, sweating, tachycardia and hypertension, often do not accompany chronic pain. There is a difference of opinion regarding the time frame for the definition, but most pain experts agree that pain lasting longer than six months is defined as chronic. $\mathrm{CNCP}$ is believed to serve no inherent biological function. Even with the clinical diagnostic 'tools' available today, we are still limited in our ability to localize precisely and define exactly the mechanism of many types of chronic pain. Pain research is discovering structural and neurochemical mechanisms in the central and peripheral nervous systems that both augment and maintain pain signal transmission in chronic pain syndromes. Thus, in many cases, chronic pain is not simply a symptom of an underlying disease process that can be treated with the expectation that the pain will disappear.

\section{II) The need for a Canadian Pain Society consensus}

1: In the past several years, there has been growing recognition on the part of health care providers, government regulators and the public that the undertreatment of pain is a major societal problem (1). Despite numerous published guidelines, current literature continues to document that acute pain, such as postoperative pain and pain in the emergency department, is often poorly managed. Although there has been significant progress, cancer pain continues to be undertreated (2). Patients with acquired immunodeficiency syndrome (AIDS) also suffer from poorly recognized and undertreated pain. A major contributor to this problem of undertreatment is the reluctance of physicians to prescribe adequate opioid therapy.

2: A bioethicist writes, "To leave a person in avoidable pain and suffering should be regarded as a serious breach of fundamental human rights" (3).

3: In 1997, the Canadian Pain Society published a position statement on Pain Relief, which included the following statements: "Almost all acute and cancer pain can be relieved, and 
many patients with chronic non-malignant pain can be helped. Patients have the right to the best pain relief possible.... $[\mathrm{H}]$ ealth professionals need to understand pain management strategies, including non-pharmacological techniques and the appropriate use of opioids" (4).

4: Until recently, opinion regarding the appropriate use of opioid analgesics in the management of CNCP was not as clear as in the case of acute pain and cancer pain. The College of Physicians and Surgeons of Alberta $(5,6)$ and the Probationary Section of Pain Physicians of the Ontario Medical Association (7) have each published guidelines supporting the appropriate use of opioids for the treatment of CNCP. The American Pain Society (APS) and the American Academy of Pain Medicine (AAPM) likewise published a joint national consensus statement in 1997 (8). However, the latter statement has many elements that are not applicable to the Canadian health care environment. As a national organization of pain professionals, it is appropriate that the Canadian Pain Society publish a Canadian consensus statement on this evolving area of medicine. It is hoped that this document, which combines and updates elements of previously published guidelines, will provide guidance for physicians, other health care providers and regulators across the country.

\section{III) Treatment options for CNCP}

1: Chronic pain can have multiple causes and a myriad of perpetuating factors. Therefore, the optimal management involves a comprehensive assessment leading to an individualized treatment approach that uses a combination of treatment options and takes into account the local availability of pain treatment resources.

2: Many strategies and options exist to treat CNCP. These include, but are not restricted to, active modalities such as stretching, therapeutic exercise, stress management skills, biofeedback and cognitive-behavioural approaches; passive modalities such as massage, manipulation, nerve and trigger point injections, and transcutaneous electronic nerve stimulation; pharmacotherapy, including nonopioid analgesics as well as opioids; and palliative surgical procedures such as implanted dorsal column stimulators, implantable drug delivery pumps and neurodestructive procedures.

3: When a specific, curative treatment exists for a given pain problem, with good evidence for effectiveness, it should always be offered first to the patient. In the absence of a specific curative therapy, it makes sense to try treatment options along an orderly continuum from least invasive, with the lowest risk of adverse effects and the best evidence for effectiveness, to treatments that are more invasive, carry a higher risk of serious adverse effects and have less evidence for effectiveness.

4: Because of the above principle, opioid analgesics are not currently recommended as first-line therapy in the treatment of most types of CNCP. However, they are a valid treatment option in patients who have failed to respond to a reasonable trial of other standard treatment modalities. In the treatment continuum approach, a trial of titrated opioid therapy should usually be offered before a recommendation for destructive palliative pain procedures.

5: Children and adolescents respond to opioids for cancer and postoperative pain very similarly to the way adults do. Children and adolescents can also suffer from conditions causing chronic nociceptive and neuropathic pain, although this type of pain is more common in adults. On the other hand, chronic pain in the elderly population is very common and often is under-recognized and undertreated. The principles of this consensus statement, with minor modifications, should be equally applicable to the pediatric and geriatric populations.

\section{IV) What is the evidence for and against the use of opioid therapy for CNCP, and how has it influenced professional opinion?}

1: Before the early 1980s, there was widespread belief that opioid analgesics were not indicated for the treatment of CNCP. Surveys originating in multidisciplinary pain programs suggested that the regular use of opioid analgesics could lead to greater psychological stress, impaired cognition and poor outcomes (9). As a result, considerable concern was voiced by opinion leaders in the medical literature that the use of opioid analgesics in the management of chronic pain would be ineffective and likely harm the patient by causing cognitive and physical impairment and addiction. These studies reported on highly selected patients using short acting opioids on a PRN basis and did not account for the impact on function of the frequent use of sedatives among their subjects. Recent empirical observation and controlled trials have challenged these opinions with evidence that opioids can sometimes provide significant relief in noncancer pain and result in functional restoration with a low risk of serious adverse effects (10).

2: Since the early 1980s, a growing number of retrospective case reports have indicated that properly selected and monitored patients with chronic pain can benefit from the use of long term opioid therapy with few adverse effects and a very low risk of addiction (10). It is recognized that such case series can be biased and lack the power of randomized controlled studies. These case series, however, challenge the conventional view that long term opioid therapy inevitably causes harm to patients. Furthermore, these studies suggest that there are very few types of chronic pain for which the prescribing of chronic opioid therapy would be contraindicated.

3: There are presently three published randomized controlled trials addressing the efficacy of scheduled oral opioids in CNCP. Two of these three Canadian trials reported on musculoskeletal pain, while the third examined opioids in neuropathic pain. One group reported on 30 patients who were 
treated for one week with sustained-release codeine or placebo in a crossover study (11). Those researchers found that patients given a mean daily codeine dose of $273 \mathrm{mg}$ experienced a $29 \%$ overall reduction in pain intensity and a $29 \%$ reduction in Pain Disability Index scores compared with a group given an as required codeine product. However, virtually all of the patients had previously used opioids for a mean duration of six years. The short duration of this study also limits its applicability to clinical practice.

Another group conducted a double-blind crossover trial in which 46 patients who had not responded to codeine, antiinflammatory agents or antidepressants were randomized to receive sustained-release morphine, up to a maximal dose of $120 \mathrm{mg}$ daily, or active placebo (benztropine) for nine weeks (12). The mean daily dose of morphine used in this study was $83.5 \mathrm{mg}$. The morphine group showed a significant reduction in pain intensity relative to that in the placebo group, but the benefit was modest - in the range of $15 \%$ to $20 \%$. There were no significant differences between the two groups in psychological features, disability status or cognition, and there was no evidence of psychological dependence or addiction. The modest reduction in pain intensity with morphine may be related, in part, to the fact that the depression scores of the participants on standard psychological tests were almost 2 SDs above the mean of a control population, although apparently representative of patients attending other tertiary pain clinics (13). Patients derived from community-based clinics may harbour less psychological distress and may show a better response to morphine or other opioid analgesics. Some patients may respond to a higher dose of opioid, although this response would be a challenge to prove in the context of a randomized controlled trial of limited duration.

Using a randomized, double-blind placebo controlled two-way crossover design, a third study evaluated controlled-release oxycodone versus placebo in patients with postherpetic neuralgia. The starting dose of controlledrelease oxycodone was $10 \mathrm{mg}$ every $12 \mathrm{~h}$, and was increased weekly up to a maximum of $30 \mathrm{mg}$ every $12 \mathrm{~h}$ over four weeks. Opioid analgesics were withdrawn before the study, but patients were permitted to continue using antidepressants and nonopioid analgesics that had been started three or more weeks before the study. Compared with patients on placebo, patients receiving controlled-release oxycodone reported statistically significant decreases in steady pain, brief (lancinating) pain and skin pain. Reductions in pain-related disability were also noted. There were no significant differences between the two groups in scores in the Beck Depression Inventory and Profile of Mood States (14).

More clinical trials need to be carried out to define populations of patients that are most likely to benefit from long term opioid therapy. Ideally, future trial designs need to take into account the spectrum of type and severity of clinical pain problems as well as the large interindividual variability in opioid pharmacodynamics. In the meantime, clinicians need to incorporate the evolving evidence with their own experience and clinical judgement when considering opioid therapy for patients with CNCP.
4: In 1993, the College of Physicians and Surgeons of Alberta became the first professional licensing body in North America to publish guidelines for opioid use in chronic nonmalignant pain $(5,6)$. These guidelines were the first in North America to endorse the use of long term opioid analgesics as an acceptable option for treating CNCP. Since that time, the College of Physicians and Surgeons of Saskatchewan and British Columbia have accepted these 'Alberta Guidelines'.

5: In the United States, an increasing number of medical jurisdictions have published opioid therapy guidelines of their own. In March 1997, the AAPM and the APS published a joint consensus statement entitled "The use of opioids for the treatment of chronic pain" (8). This statement accepted the use of opioids for chronic pain as "legitimate medical therapy". At its annual meeting in May 1997, the Canadian Pain Society adopted a resolution accepting the principles of the AAPM/APS consensus statement along with a commitment to adapt them for the Canadian health care environment. In Fall 1997, a committee of the Probationary Section on Chronic Pain of the Ontario Medical Association, published a discussion paper which reviewed the above two sets of guidelines on this topic (7).

6: In a 1997 public policy statement on the rights and responsibilities of physicians prescribing opioid analgesics for the treatment of pain, the American Society of Addiction Medicine (ASAM) made a series of recommendations supporting the use of opioids for chronic pain providing there is appropriate assessment and monitoring (15).

7: This Canadian Pain Society consensus statement includes elements from all of the above sources and acknowledges their contribution. This document does not in any way sanction the inappropriate prescribing of opioid analgesics, nor does it endorse opioid therapy as the only treatment modality for chronic pain. It recognizes that the prescribing of long term opioid therapy is considered controversial by some physicians. It is intended, however, as a means to raise awareness and to assist both Canadian physicians and Canadian regulators in understanding the role of opioid analgesics in the treatment of CNCP.

\section{V) Many commonly held assumptions regarding opioids and CNCP need to be revised in light of new information and recent clinical experience}

1 - Addiction: Misinterpreting the efforts of patients to get relief from their pain as addiction or drug seeking behaviour can result in stigmatization and unnecessary withholding of opioid analgesics. Clinicians as well as regulators can mistake physical dependence with addiction. Addiction is a biopsychosocial disorder characterized by the compulsive use of a substance and preoccupation with obtaining it, despite evidence that its continued use results in physical, emotional, social or economic harm. In a public policy statement released in 1997, The American Society of Addiction Medicine (ASAM) defined addiction in the context of pain treatment with opioids as "a persistent pattern of dysfunctional opioid 
use that may involve any or all of the following: adverse consequences associated with the use of opioids; loss of control over the use of opioids; preoccupation with obtaining opioids, despite the presence of adequate analgesia" (16).

Studies have shown that the development of iatrogenic addiction is rare when opioids are carefully prescribed for the relief of acute or cancer pain. Older literature from chronic pain clinics reporting high rates of opioid abuse and addiction used nonstandardized diagnostic criteria $(17,18)$. Careful screening of patients for risk factors may further reduce the possibility of unrecognized iatrogenic opioid addiction. In addition, evidence is emerging that patients with addiction disorders who also suffer from CNCP might benefit from the judicious use of opioid analgesics when prescribed with appropriate caution $(19,20)$.

2 - Tolerance: Tolerance to the adverse effects of opioid analgesics, such as somnolence and nausea, appears to develop readily and is a welcome clinical phenomenon. On the other hand, analgesic tolerance occurs when progressively higher doses of opioids are required to maintain pain control. This was previously thought to be a universal occurrence and, therefore, limit the efficacy of opioids on a long term basis. Experience with cancer patients has shown that analgesic tolerance is rarely the driving force for dose escalation when opioids are dosed to effect. An increase in the analgesic requirements of cancer pain patients is usually due to progression of the patient's disease. Retrospective case reports suggest that the development of analgesic tolerance in chronic pain patients is also uncommon and is most relevant within the first six months of opioid use. Furthermore, when physiological tolerance occurs, it does not preclude further achievement of adequate analgesia. Tolerance is one of the criteria for opioid addiction that is listed in the Diagnostic and Statistical Manual of Mental Disorders, Fourth Edition (DSM-IV) (21). However, according to the ASAM public policy statement, tolerance "does not, in and of itself, imply addiction" (16). It should, therefore, not be used to diagnose addiction in the absence of other criteria listed above.

3 - Physical dependence: Physical dependence is a common physiological phenomenon characterized by the appearance of a constellation of signs and symptoms associated with the abrupt termination of regular opioid use. Common symptoms include coryza, tremors, sweats, chills, lacrimation, abdominal cramps, arthralgias and myalgias, vomiting and diarrhea. Physical dependence, in the absence of other indicators, is neither predictive nor diagnostic of addiction. Most cancer patients on scheduled opioid therapy become physically dependent. Although abrupt withdrawal of opioid therapy is not life-threatening, it is rarely necessary in the skilled and sensitive treatment of patients. If the need to discontinue long term opioid therapy arises, then withdrawal symptoms can be minimized by gradual tapering of opioid therapy and the use of adjunctive medication to mute the abstinence syndrome.
4 - Opioid dosage: Patients exhibit tremendous interindividual variability with respect to the pharmacokinetics, pharmacodynamics and side effect profile of a given opioid. This variability may involve differences in receptor type sensitivity, metabolic pathways or other as yet unknown genetic factors. The main two generalizations are that elderly patients usually require a lower dose to achieve effective pain relief than younger patients and that neuropathic pain usually requires higher opioid doses than nociceptive pain. The principle used in all types of pain management with opioids is known as 'dosing to effect'. Opioid analgesics should be started at a low dose and carefully titrated until an adequate level of analgesia is obtained, or until persistent and unacceptable side effects warrant a reevaluation of therapy. Failure to realize at least partial analgesia with incremental dosing in the opioid-naive patient may indicate that the pain syndrome is unresponsive to opioid therapy (see 'Opioid responsiveness' below). Recent anecdotal experience suggests that, for some patients with chronic pain, opioids do not exert an appreciable analgesic effect until a threshold dose has been achieved. There is, however, no controlled clinical data addressing either of the above observations.

5 - Side effects: There is no recorded risk in the medical literature of direct permanent organ damage with the long term clinical use of opioid therapy. This is in contrast to most other classes of analgesics in use today. Fear of causing respiratory depression has often been invoked as a rationale for not using opioids to treat pain. It is now widely accepted that respiratory depression caused by opioid analgesics tends to occur largely in opioid-naive patients. It is a short-lived phenomenon that tends to be antagonized by pain. In CNCP, the risk of respiratory depression with oral dosing is extremely low and can be further minimized by careful titration of the dosage of opioids to the level of pain. Constipation commonly accompanies opioid therapy and is usually harder to treat than to prevent. It is, therefore, important to manage this side effect prophylactically using a stepped approach involving adequate dietary fibre, stool softeners, osmotic agents and, if necessary, regular stimulant laxative use. Nausea is a common early side effect of regular opioid therapy, and usually resolves with continued use. Antinauseants may be recommended during the initial titration phase. Sedation and cognitive deficits are also early side effects for which tolerance with continued use frequently develops once stable dosing has been achieved. There is little current evidence that the long term use of scheduled, stable dose opioid therapy leads to clinically significant cognitive or psychomotor deficits in patients with chronic pain. Recent evidence suggests that pain itself can have an adverse effect on cognitive performance, which is improved with opioid analgesia $(22,23)$. The longest clinical noncancer experience with patients on opioids is over 30 years of continuous use in the methadone maintained population of opioid addicts. Studies of this population have shown no organ toxicity and no increase in markers of cognitive dysfunction such as motor vehicle accidents or infractions of driving codes. A recent study 
comparing cancer patients on stable, long term, opioid therapy with a group on no opioids, demonstrated no significant difference in functions related to driving ability $(24,25)$. Cognitive deficits in opioid-treated patients are more often due to the concurrent use of sedative medications such as benzodiazepines. Therefore, use of sedatives in patients on long term opioid therapy should be avoided where possible.

6 - Opioid responsiveness: Pain specialists previously believed that certain types of pain, such as neuropathic pain, were 'resistant' to opioid analgesics. This opinion was challenged by subsequent research that demonstrated that neuropathic pain can respond to opioids but at relatively higher doses. The opioid responsiveness of a given pain syndrome in a particular patient refers to the balance of analgesia versus adverse effects. It is a dynamic process over time, which can be affected by factors such as the type of pain, the physiology of the particular patient and the characteristics of the particular opioid. For some types of pain in certain patients, a given opioid can provide very effective analgesia at low doses, with minimal adverse effects. For other pain syndromes, higher doses of opioids may be required, which, in a particular patient, may result in unacceptable persistent side effects, even with careful titration. Early understanding of opioid pharmacology assumed that all opioid analgesics shared the same mechanism of action and were thus completely interchangeable. Clinical and experimental evidence is now evolving of interindividual variability in responsiveness to the different opioids - possibly due to differential opioid receptor stimulation or other as yet unknown factors. For example, morphine, the prototype mu receptor agonist, may cause more side effects than analgesia in a given patient than levorphanol (not available in Canada except on special release), or oxycodone, which appear to have both mu and kappa agonist activity, or methadone, a mu agonist with N-Methyl-D-Aspartate receptor blocking properties. Therefore, before labelling a patient in pain as 'unresponsive to opioids', clinicians should consider the following:

i) provide an adequate therapeutic trial by dosing to effect;

ii) allow adequate time during titration for tolerance to adverse effects to develop;

iii) demonstrate a consistently unfavourable balance of intolerable adverse effects to analgesia; and

iv) duplicate this in a sequential trial of opioid analgesics with different properties.

Patients whose pain is found to be unresponsive to an adequate trial of opioid therapy should not be assumed to have psychogenic pain or to be malingering.
7 - Diversion: Preventing the diversion of opioid analgesics for illicit use is the concern of every conscientious prescriber, but strategies to discourage diversion should not take precedence over effective pain management. The risk of diversion can be reduced when prescribers practise with an awareness of the characteristic patterns of drug diversion and drugseeking patients. According to the ASAM public policy statement, "physicians who are practising medicine in good faith and who use reasonable medical judgment regarding the prescription of opioids for the treatment of pain should not be held responsible for the willful and deceptive behaviour of patients who successfully obtain opioids for nonmedical purposes" (15).

\section{VI) Knowledge is evolving}

1: Ongoing research is slowly unravelling the mysteries of chronic pain. As new information becomes available, it is expected that consensus statements such as these will be revised appropriately. Physicians who prescribe long term opioid therapy to patients with chronic noncancer pain are encouraged to stay abreast of these developments by reading relevant peerreviewed literature and by attending continuing medical education courses.

2: As clinical knowledge advances, it is hoped that treatments will increasingly become available that specifically remove or correct the underlying cause of many pain syndromes. However, until this is possible, the treatment of the pain, with whatever modality is most effective, is a necessary part of medical practice, subject to the need to take toxicity, side effects, patient preference and availability (including cost) into account.

\section{VII) Accepted principles of practice for the use of opioid analgesics should be promulgated}

1: It is clear that physicians, other health care providers, government regulators and law enforcement agencies seek guidance from each other or seek consensus through discussion regarding the appropriate use of opioid analgesics in the treatment of CNCP. Regulators and law enforcement authorities are charged with the dual responsibilities of preventing drug diversion without interfering with the appropriate medical use of opioid analgesics. As such, they require guidelines that establish the use of opioids to treat chronic pain as a legitimate medical practice. At the same time, prescribers have been shown to be reluctant to prescribe opioid analgesics because of fear of regulatory scrutiny. Guidelines may help to alleviate the regulatory concerns of prescribers, thus increasing the availability of opioid analgesics to chronic pain sufferers.

2: The Canadian Pain Society believes that guidelines for prescribing long term opioid therapy should be an extension of the basic principles of good medical practice. It is hoped that organizations developing clinical practice guidelines will make use of the following principles. 


\section{GENERAL PRINCIPLES OF APPROPRIATE PAIN MANAGEMENT WITH OPIOIDS}

\section{Evaluation of the patient}

1: Each patient with CNCP should be thoroughly evaluated before the institution of long term opioid therapy. If possible, the specific cause of the pain should be determined, and specific therapy, if available, should be offered. Evaluation of the patient should include at least the following information:

i) a detailed pain history and the results of previous treatments;

ii) assessment of the impact of pain on the patient's family or significant others;

iii) a directed physical examination, including musculoskeletal examination, to look for clues to specific pain syndromes;

iv) a review of previous diagnostic studies and assessments. Additional investigation or consultation, if required, to fill in gaps in the previous diagnostic work-up:

v) an assessment of coexisting illnesses and treatments, and their effect on the patient and on the pain; and

vi) an assessment of significant psychological, social or behavioural factors that may affect the current pain problem or future treatment plans. This includes an assessment of risk factors for addiction.

2: A complete assessment of the pain problem must precede the initiation of a trial of opioid therapy, but this does not require the duplication of previous investigations or consultations.

3: When a specific curable pain syndrome cannot be diagnosed, it may be useful, both for research purposes and clinically, to classify the type of pain into three main categories based on the inferred pathophysiology.

Troublesome nociceptive pain is usually due to continuous stimulation of specialized pain receptors in such tissues as the skin, bones, joints and viscera. It is often indicative of ongoing tissue damage. Typical examples include osteoarthritis and chronic pancreatitis.

Neuropathic pain is due to nerve damage or abnormal processing of signals along the pain systems of the peripheral and central nervous system. Examples include postherpetic neuralgia, phantom limb pain, pain resulting from spinal cord injuries and sympathetically mediated pain. Most chronic pain syndromes involve one or both of the above mechanisms. Occasionally, a patient's pain pattern may not precisely fit into either of the above categories. Previous guidelines have labelled this type of pain as idiopathic. As knowledge evolves regarding central and peripheral sensitization of the central nervous system pain systems it is likely that many types of idiopathic pain will turn out to have a nociceptive or neuropathic origin or a mixture of the two.

4: Nociceptive and neuropathic pain syndromes can both be considered for a trial of opioid therapy. Patients with neuropathic pain may require higher doses of opioid therapy to achieve significant analgesia and may benefit from the concurrent use of adjuvant analgesics from the tricyclic antidepressant, anticonvulsant or antiarrhythmic classes. Idiopathic pain may also be an indication for opioid therapy, but previous guidelines have suggested that a trial of opioids be prescribed cautiously with specific goals and careful monitoring to document an ongoing benefit.

5: All types of pain may have a significant affective or psychological component. Depressive symptoms frequently accompany $\mathrm{CNCP}$ and contribute to patient suffering. It is generally accepted by pain specialists that depression is more likely to be a secondary effect of the pain itself, rather than the reverse. Some patients with chronic pain and symptoms of major depression may demonstrate decreased suffering when depression is treated. For others, depressive symptoms diminish when pain is adequately treated. The term 'psychogenic pain' has been used to define pain that is believed to be caused by or primarily influenced by a psychopathological process. The use of this term is discouraged because it lacks precision and has the potential to stigmatize patients when applied inappropriately. True primary psychological pain disorders are rare (26) and should be classified using criteria of the International Association for the Study of Pain or the DSM-IV $(27,21)$.

6: An important component of the psychosocial evaluation for opioid therapy is the assessment of the risk of addiction. Because an unrecognized addictive disorder can complicate the treatment of chronic pain, it is worthwhile to screen patients to identify those who may need more detailed assessment. A basic, suggested set of screening questions is included as Appendix 1 to this document. Patients with a past history of addiction should not necessarily be denied a trial of opioid therapy, but will require more careful prescribing and follow-up.

7 - Treatment plan: The treatment plan should be individualized to the patient and to the pain problem. The physician should consider the gamut of appropriate treatment approaches, including physical methods, multidisciplinary pain management programs, cognitive and behavioural strategies, 
pharmacotherapy, and various other invasive and noninvasive techniques. The choice may depend on many factors such as cost, availability of timely services and comorbidity, as well as physical and psychosocial impairments related to the pain. For some patients, simply decreasing the severity of their pain is all that is required to improve their quality of life. For others, a more intensive comprehensive treatment plan that addresses the psychological, social and behavioural contributors to their suffering is required.

8: In most circumstances, a trial of scheduled long term opioid therapy may be indicated for patients who have failed to respond to a reasonable documented trial of nonpharmacological and nonopioid pharmacological modalities.

9: The primary purpose of long term opioid therapy should be improved quality of life for the patient. Therefore, improved pain control is a reasonable and appropriate goal of treatment. In CNCP it is usually not realistic to set a goal of total elimination of the pain. Instead, the patient and physician need to negotiate a treatment plan to find the optimum balance of pain relief, functional improvement and medication side effects. To help patients improve their level of physical and psychological function, it is often useful to develop with the patient a list of functional goals. These goals might include specific targets for physical activity, performance of activities of daily living, hobbies or return to work. The attainment of these goals can be used as evidence of the efficacy of long term opioid therapy. However, failure to achieve fully all functional goals should not necessarily be construed as a therapeutic failure. On the other hand, a persistent decline in physical or psychological function in association with institution of opioid therapy should cause the physician to reassess carefully the benefits of ongoing treatment with opioids. In some cases, a gradual dose reduction possibly leading to discontinuation of opioid therapy may be required.

10: If a trial of opioid analgesics is selected, the physician should obtain informed consent from the patient or the patient's guardian. Informed consent should include discussion of the risks and benefits of opioid therapy, as well as the conditions under which opioids will be prescribed. A suggested list of discussion points is included in Appendix 2. In most practice settings in Canada a documented verbal consent will usually suffice. For patients assessed to be at higher risk of noncompliance with the agreed upon treatment plan, physicians may find it helpful to use a written therapeutic agreement, setting out the terms and conditions for prescribing opioid therapy. A sample blank agreement has been included in Appendix 3.

11: When prescribing an opioid analgesic for around-theclock pain, it should also be dosed around-the-clock in a pharmacologically appropriate, time-contingent, dosing schedule. There is no pharmacological rationale for a dose ceiling for opioids. Long term opioid therapy should be started at a low dose and carefully titrated until an adequate level of analgesia is obtained, or until unmanageable and persistent side effects warrant a decreased dose or a change in therapy. For opioid-naive patients, failure to realize at least partial analgesia with incremental dosing may indicate a pain syndrome that is less responsive to opioids. For some patients, however, opioids do not exert an appreciable analgesic effect until a threshold dose has been achieved. Use of an opioid with a long duration of action has many advantages for treating chronic pain. It can facilitate patient compliance with around-the-clock dosing; can provide a more consistent blood level, thereby allowing better tolerance to side effects, such as cognitive impairment and may reduce the reinforcement of pain behaviour based on as required dosing regimens. During the titration phase, reasonable doses of breakthrough opioid may be provided and can be used to assess the adequacy of the overall opioid dose. A goal of optimal opioid titration for a stable chronic pain condition is to decrease the frequency of breakthrough doses to a minimum.

12 - Consultation as needed: Consultation with a specialist in pain medicine or with a pain psychiatrist or psychologist may be warranted, depending on the expertise of the practitioner and the complexity of the presenting problem. Consultants in pain medicine are not always available on a timely basis to primary care physicians. Therefore, a consultation with a specialist in pain management should not be a prerequisite to the use of opioid therapy. The presence of addiction or a comorbid psychiatric disorder may require co-management with a specialist in addiction medicine or a psychiatrist, respectively.

13 - Periodic review of the patient: Periodic review of the patient is an essential part of ongoing management with opioid therapy. As with the initial evaluation of the patient, reassessment of the patient's pain is based mainly on the patient's self-report. In assessing the efficacy of opioid therapy, it may be helpful to use collateral sources of information, such as family members, employers, etc. Periodic reexamination is warranted to assess the nature and evolution of the pain complaint and to ensure the ongoing benefit of opioid therapy. It is recommended that the following points be specifically covered at follow-up visits.

i) Record the patient's self-reported level of pain using some type of quantitative scale such as a Visual Analogue Scale or a verbal rating scale from 0 to 5 or from 0 to 10 .

ii) Record the level of physical and psychological function, listing specific activities where appropriate.

iii) Record any side effects of opioid therapy (such as drowsiness, nausea and vomiting, constipation and sweating) and their management.

iv) Record any suspicious drug-seeking or other aberrant behaviours observed by the physician or reported by others, along with the action taken by the physician. 
v) If writing a prescription for opioid therapy, be certain to record the name of the drug, the strength, the number of dosage units and how the drug is to be taken. Record any changes to opioid therapy and the reasons for them.

14: The adverse effects of opioid therapy may sometimes contribute to a persistent decrease in function. In some cases, a gradual reduction in the dosage of opioid therapy - possibly leading to discontinuation of therapy - may be the appropriate course of action.

15 - Documentation: Documentation is essential to demonstrate the evaluation process, including consultations and relevant investigations, the rationale for long term opioid therapy in the context of the overall management plan and the periodic review of patient status. In addition, documentation is required to demonstrate compliance with federal controlled substance legislation.

\section{REFERENCES}

1. Gureje O, Von Korff M, Simon GE, Gater R. Persistent pain and well-being. A World Health Organization study in primary care. JAMA 1998;280:147-51.

2. Bernabei R, Gambassi G, Lapane K, et al. Management of pain in elderly patients with cancer. JAMA 1998;279:1877-82.

3. Somerville MA. Opioids for chronic pain of non-malignant origin Coercion or consent? Health Care Analysis 1995;3:12-4.

4. The Canadian Pain Society. Position Statement on Pain Relief. Ottawa: Canadian Pain Society, 1997.

5. Hagen NA, Flynne P, Hays H, MacDonald N. Guidelines for the management of chronic non-malignant pain. Opioids and other agents. Can Fam Physician 1995;41:49-53.

6. The College of Physicians and Surgeons of Alberta. Guidelines for management of chronic non-malignant pain. Edmonton: College of Physicians and Surgeons of Alberta, 1993.

7. Goldman B, Gale G, Gilchrist G, Jacobs H, Kerr I, Rothbart P. Use of opioid analgesics for the treatment of chronic pain of nonmalignant origin. A discussion paper from the Probationary Section on Chronic Pain of the Ontario Medical Association. Pain Res Manage 1997;2:231-7.

8. The use of opioids for the treatment of chronic pain. A consensus statement from the American Academy of Pain Medicine and the American Pain Society. Clin J Pain 1997;13:6-8.

9. McNairy SL, Maruta T, Ivnik RJ, Swanson DW, Ilstrup DM. Prescription medication dependence and neuropsychologic function. Pain 1984;18:169-77.

10. Portenoy RK. Opioid therapy for chronic nonmalignant pain. Pain Res Manage 1996;1:17-28.
11. Arkinstall W, Sandler A, Goughnour B, Babul N, Harsanyi Z, Darke AC. Efficacy of controlled-release codeine in chronic non-malignant pain: a randomized, placebo-controlled trial. Pain 1995;62:169-78.

12. Moulin DE, Iezzi A, Amireh R, Sharpe WK, Boyd D, Merskey H. Randomised trial of oral morphine for chronic non-cancer pain. Lancet 1996;347:143-7.

13. Crook J, Tunks E. Epidemiologic comparison of persistent pain sufferers in a specialty pain clinic and in the community. Arch Phys Med Rehabil 1986;67:451-5.

14. Watson CP, Babul N. Efficacy of oxycodone in neuropathic pain: a randomized trial in postherpetic neuralgia. Neurology 1998;50:1837-41.

15. American Society of Addiction Medicine. Public policy statement on the rights and responsibilities of physicians in the use of opioids for the treatment of pain. Chevy Chase: American Society of Addiction Medicine, 1997.

16. American Society of Addiction Medicine. Public policy statement on definitions related to the use of opioids in pain treatment. Chevy Chase: American Society of Addiction Medicine, 1997.

17. Fishbain DA, Rosomoff L, Rosomoff DS. Drug abuse, dependence, and addiction in chronic pain patients. Clin J Pain 1992;8:77-85.

18. Brown RL, Patterson JJ, Rounds LA, Papasouliotis O. Substance abuse among patients with chronic back pain. J Fam Pract 1996;43:152-60.

19. Dunbar SA, Katz NP. Chronic opioid therapy for non-malignant pain in patients with a history of substance abuse: Report of 20 cases. J Pain Symptom Manage 1996;11:163-71.

20. Kennedy JA, Crowley TJ. Chronic pain and substance abuse: a pilot study of opioid maintenance. J Subst Abuse Treat 1990;7:233-8.

21. American Psychiatric Association. Diagnostic and Statistical Manual of Mental Disorders, 4rd edn, revised. Washington: American Psychiatric Association, 1994.

22. Lorenz J, Beck H, Bromm B. Cognitive performance, mood and experimental pain before and during morphine-induced analgesia in patients with chronic non-malignant pain. Pain 1997;73:369-75.

23. Haythornthwaite JA, Menefee LA. Outcome of chronic opioid therapy for non-cancer pain. J Pain Symptom Manage 1998;15:185-94.

24. Vainio A, Ollila J, Matikainen E, Rosenberg P, Kalso E. Driving ability in cancer patients receiving long-term morphine analgesia. Lancet 1995;346:667-70.

25. Zacny JP. Should people taking opioids for medical reasons be allowed to work and drive? Addiction 1996;91:1581-4.

26. Merskey H. Psychiatry and chronic pain. Can J Psychiatry 1989;34:329-36.

27. Merskey H, Bogduk N, eds. Classification of Chronic Pain: Descriptions of Chronic Pain Syndromes and Definitions of Terms. International Association for the study of Pain (IASP). Seattle: IASP Press, 1994.

APPENDICES FOLLOW ON NEXT PAGE 


\section{APPENDIX 1 \\ Suggested addiction screening questions}

In screening patients with chronic noncancer pain for addiction risk, the clinician is primarily interested in assessing for patients with a history of alcohol abuse/dependence or with a history of polydrug abuse. A patient who has a past history of abusing one substance is at higher risk for abusing other psychoactive substances. The purpose of screening is not to deny patients opioids for pain, but to identify the small subgroup at higher risk for more detailed assessment and more careful monitoring.

The Screening Instrument For Substance Abuse Potential (SISAP) is a five-item screening tool created by Coambs et al in 1996 (1) that helps the clinician to categorize patients into lower or higher risk of abusing prescribed opioids. It requires that the physician already know the patient or have collateral information to confirm the accuracy of the answers. It has a high false positive rate but a low false negative rate when tested against the database of a large $(n=11,634)$ Canadian epidemiological survey of alcohol and drug use. It has not yet been prospectively tested in the chronic pain population.

The five SISAP questions are:

1. If you drink alcohol, how many drinks do you have on a typical day?

2. How many drinks do you have in a typical week?

3. Have you used marijuana or hashish in the past year?

4. Have you ever smoked cigarettes?

5. What is your age?

Use caution when prescribing opioids for the following patients:

1. Men who exceed four drinks per day or 16 drinks per week

2. Women who exceed three drinks per day or 12 drinks per week

3. A patient who admits to marijuana or hashish use in the past year. (It is recreational use of cannabis for euphoric effect that is of concern. The use of tetrahydrocannabinol (THC) derivatives to treat pain is still very controversial. Clinicians should exercise caution in recommending opioid therapy to a patient who is using cannabis regularly.)

4. A patient under 40 years who smokes.

The majority of patients will pass the screen and are probably at low risk of abusing opioids, but clinical judgement is still required. The SISAP questions ask about recent drug or alcohol use and may, therefore, miss a patient who is at risk because of a previous history of chemical abuse or dependency. A simple but effective question to ask is:

Has your use of alcohol or other drugs ever caused a problem for you or those close to you?

A positive answer to the above or to any of the SISAP questions suggests further assessment.

The CAGE-AID questions comprise a quick screening tool to assess for the risk of serious alcohol or drug problems.

In the past have you ever:

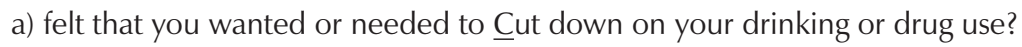

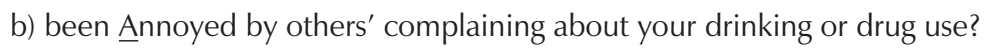

c) felt Guilty about the consequences of your drinking or drug use?

d) had a drink or taken a drug in the morning (Eye-opener) to decrease hangover or withdrawal symptoms?

One positive response to any one of the CAGE-AID questions should raise concerns. Two or more positive responses means a high likelihood of a serious alcohol or drug problem and may require a formal addiction assessment by a specialist.

A family history of alcohol, drug abuse or significant psychiatric illness, or a personal history of previous physical, sexual or emotional abuse may also be risk factors for substance abuse and require assessment.

\section{REFERENCE}

1. Coambs RB, Jarry JL, Santhiapillai AC, Abrahamsohn RV, Atance CM. The SISAP: A new screening instrument for identifying potential opioid abusers in the management of chronic nonmalignant pain in general medical practice. Pain Res Manage 1996;1:155-62. 


\section{APPENDIX 2}

Discussing opioid therapy with the patient - suggested points of discussion

1. Describe and explain the purpose of opioid therapy (less pain rather than no pain) with the patient and/or guardian, along with explaining the common side effects and their management. Preventative management of constipation should specifically be discussed. The small risk of addiction in low risk patients should be addressed and differentiated from tolerance and physical dependence. Warn the patient regarding withdrawal symptoms due to abrupt discontinuation of opioids. Discuss the concept of dose titration and the importance of time-contingent dosing versus as required dosing for around-the-clock pain. Discuss the appropriate use of breakthrough medication.

2. Advise the patient and/or guardian that drowsiness is a common side effect during titration of opioid therapy. The patient should not drive a car or operate dangerous machinery until this phase of drowsiness has passed. Failure to comply with this advice may result in a duty to report to the provincial Ministry of Transportation.

3. The patient and/or guardian should be warned not to change the dosage of opioid analgesic nor the dosing interval without specific instructions from the doctor. The patient should be made aware that such unsanctioned dosage changes may compromise the physician-patient relationship.

4. Inform the patient and/or guardian that regular follow-up appointments are required to monitor the effectiveness of opioid treatment and to manage side effects. The frequency of follow-up appointments will vary depending on the phase of treatment - titration versus stable dosing.

5. Inform the patient and/or guardian that prescriptions for opioid analgesics should be obtained only from one physician or, in the absence of that physician, his or her designate. The patient should have all prescriptions for psychoactive medication dispensed at one pharmacy, except in emergencies. Inform the patient and/or guardian that seeking opioid treatment from other physicians and pharmacies without informing the prescribing physician undermines the trust essential to prescribing long term opioid therapy.

6. Advise the patient and/or guardian to keep the opioid analgesics in a safe and secure place, and to not give, lend or sell the medication to anyone.

7. Warn the patient and/or guardian that there is a potential for significant cognitive dysfunction if opioids are combined with sedatives such as benzodiazepines, barbiturates, muscle relaxants, or alcohol. The patient and/or guardian should be warned not to consume any of the above substances without first discussing this with the physician.

8. Although the potential for abuse or addiction to prescribed opioid analgesics is small in low risk patients, the concurrent abuse of illicit substances such as marijuana, cocaine, stimulants, hallucinogens, heroin or the consumption of alcohol in a high risk pattern identifies an individual at increased risk of also abusing opioids. The use of these substances may also interfere with the therapeutic effect of opioids or cause increased side effects such as cognitive dysfunction. It is therefore advisable that the patient abstain from taking any psychoactive substances without first discussing this with the physician. Advise the patient and/or guardian that the physician may, from time to time, take specific actions to monitor for this possibility such as periodic blood and/or urine drug screening. This may also include an assessment with a specialist in addiction medicine.

9. Inform the patient and/or guardian that, as part of ongoing treatment, the physician may request additional consultations and assessments, or recommend other concurrent treatment modalities. The clinician should carefully re-evaluate a patient who consistently refuses to cooperate with recommendations for treatments other than opioid therapy.

10. Inform the patient and/or guardian that, aside from better pain control, a key measure of the efficacy of long term opioid therapy is improved physical and psychological function at home and/or work. The patient and the physician may, therefore, discuss a set of reasonable specific functional goals. The physician will assess progress towards these goals at each visit and will use this information in evaluating the overall success of long term opioid therapy. Persistent functional decline on opioids may result in re-evaluation of the patient and a reassessment of the treatment plan. 


\section{APPENDIX 3}

Sample basic patient agreement (for patients at higher risk of noncompliance with opioid therapy)

1. I, agree that Dr will be the only physician prescribing OPIOID (also known as NARCOTIC) pain medication.

2. I will take the medication at the dose and frequency prescribed by my physician. I agree not to increase the dose of opioid on my own and understand that doing so may lead to the treatment with opioids being stopped.

3. I will attend all appointments, treatments and consultations as requested by my physician.

4. I will not receive opioid pain medications from any other physician except in an emergency or in the unlikely event that I run out of medication. Should such occasions occur, I will inform my prescribing physician as soon as possible.

5. I understand that the common side effects of opioid therapy include nausea, constipation, sweating and itchiness of the skin. Drowsiness may occur when starting opioid therapy or when increasing the dosage. I agree to refrain from driving a motor vehicle or operating dangerous machinery until such drowsiness disappears.

6. I understand that there is small risk that I may become addicted to the opioids I am being prescribed. As such, my physician may require that I have additional tests and/or see a specialist in addiction should a concern about addiction arise during my treatment.

7. I understand that the use of any mood-modifying substance, such as tranquilizers, sleeping pills, alcohol or illicit drugs (such as cannabis, cocaine, heroin or hallucinogens), can cause adverse effects or interfere with opioid therapy. Therefore, I agree to refrain from the use of all of these substances without first discussing it with my physician.

8. I agree to be responsible for the secure storage of my medication at all times. I agree not to provide my prescribed pain medication to any other person.

9. If I break this agreement, my physician reserves the right to stop prescribing opioid medications for me.

10. I hereby agree that my physician has the authority to disclose the prescribing information in my patient file to other health care professionals when it is deemed medically necessary in the physician's judgement. 


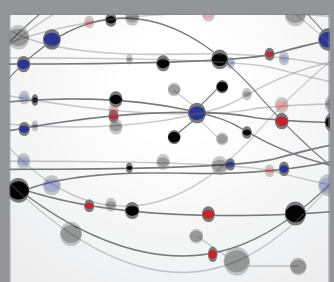

The Scientific World Journal
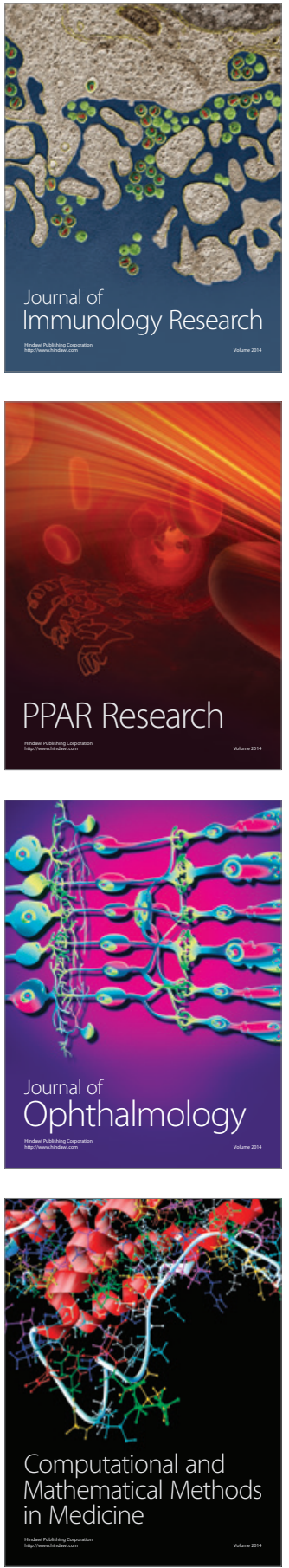

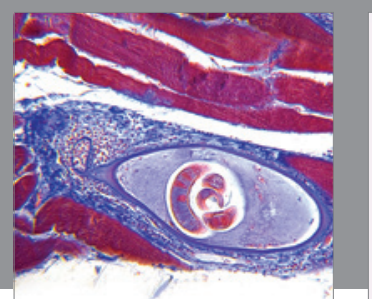

Gastroenterology Research and Practice

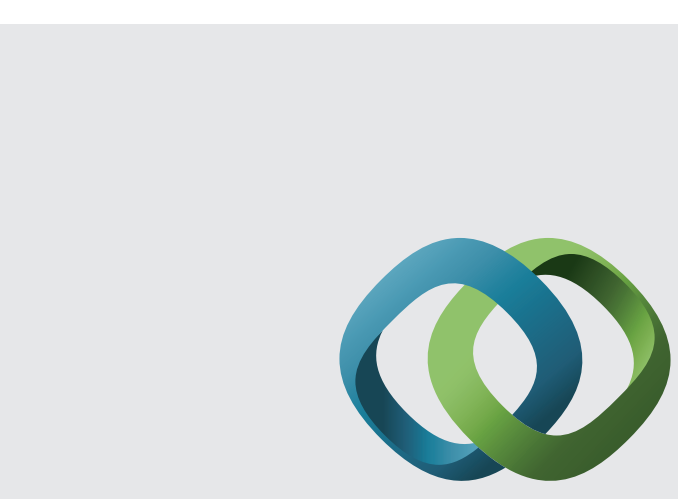

\section{Hindawi}

Submit your manuscripts at

http://www.hindawi.com
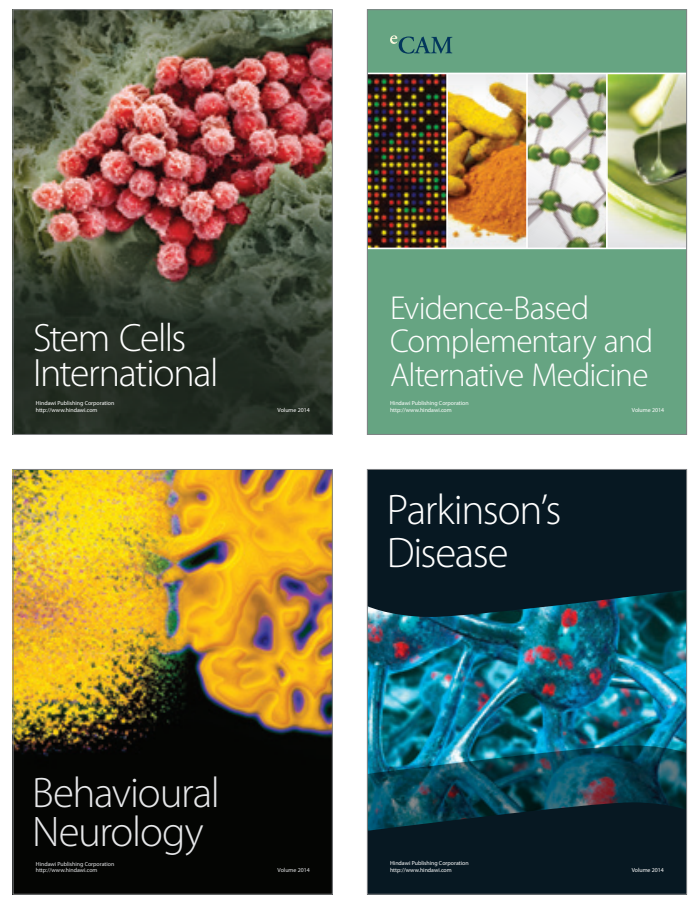
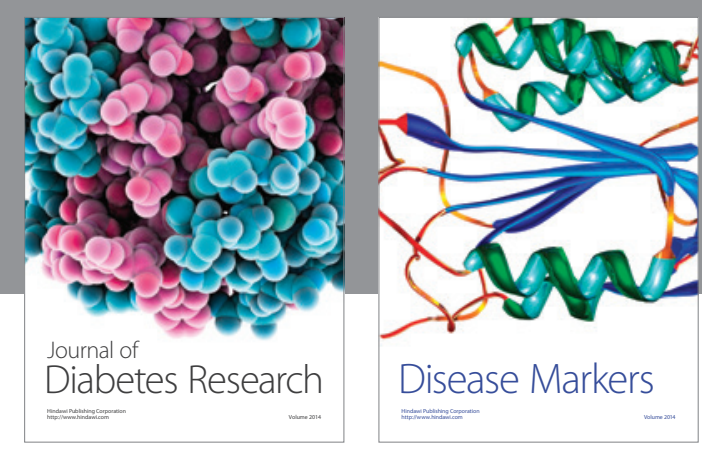

Disease Markers
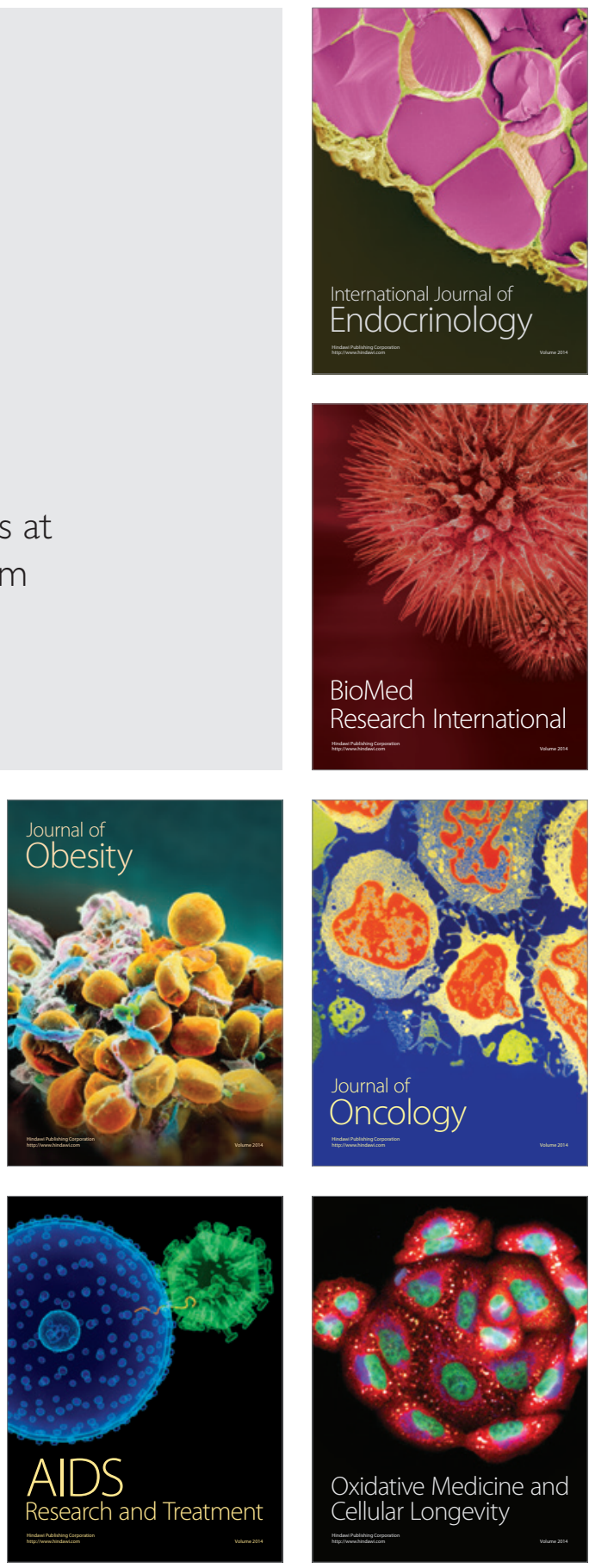